\title{
Lipoid Proteinosis presenting as beaded papules of the eyelid: report of three cases
}

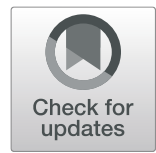

Zhenyu Wei ${ }^{1}$, Antoine Labbe ${ }^{1,2,3,4}$ and Qingfeng Liang ${ }^{*}$

\begin{abstract}
Background: Lipoid proteinosis (LP) is a rare multisystem inherited disease. We report here in three LP cases with beaded papules of the eyelid. Their clinical presentations, histological characteristics, and genetic findings are described and discussed.

Case presentation: A 12-year-old boy reported to our hospital with a complaint of ocular irritation, redness, and tearing for two years. He had a history of hoarseness since childhood. His younger brother (5 years old) also complained of hoarseness. Another patient, a 26-year-old woman, described many beaded papules on the edge of her eyelids since age 11 years. She additionally reported hoarseness since 4 years of age. Careful slit-lamp examination in these cases revealed waxy beaded papules on the margins of both eyelids and mild conjunctival congestion. Physical examination showed irregular, rugged scars on their facial skin. Genetic analysis showed the mutation located in exon 6 of the ECM1 gene.

Conclusions: Three LP cases first diagnosed by ophthalmologists are presented. The presence of eyelid papules should prompt the ophthalmologist to pay close attention to the patient's voice. If there is a definite history of hoarseness, these patients should undergo gene sequence analysis. If necessary, otorhinolaryngology and dermatology consults may help confirm the diagnosis. Treatment is primarily symptomatic to improve patients' quality of life.
\end{abstract}

Keywords: Case report, Lipoid proteinosis, Eyelid disease, Extracellular matrix gene 1

\section{Background}

Lipoid proteinosis (LP), first described by Urbach and Wiethe in 1929, is an autosomal recessive genodermatosis caused by mutations in the extracellular matrix gene 1 (ECM1) on chromosome 1q21 [1]. LP varies in clinical manifestations and severity, usually presenting as hoarseness in early childhood, with subsequent mucocutaneous lesions [2]. Neurological complications have been also described [2]. Here, we report three cases of LP patients with beaded papules of the eyelid, along with oropharyngeal and skin manifestations.

\footnotetext{
* Correspondence: lqflucky@163.com

${ }^{1}$ Beijing Key Laboratory of Ophthalmology and Visual Sciences, Beijing Institute of Ophthalmology, Beijing Tongren Eye Center, Beijing Tongren Hospital, Capital Medical University, 100005 Beijing, China

Full list of author information is available at the end of the article
}

\section{Case presentation}

Case 1-2

A 12-year-old boy (case 1) was referred to our department with complaints of ocular irritation, redness, and tearing for 2 years. He had a history of hoarseness since childhood and complained of recurrent large ulcerations on his tongue, as well as fragile skin with bullous lesions that appeared after minimal trauma. His younger brother (5 years old, case 2) had no complaints other than hoarseness. They all denied a previous history of surgery or medication. Their parents and close relatives had no similar history.

Slit-lamp examination revealed waxy beaded papules on the margins of both eyelids and mild conjunctival congestion in both cases (Fig. 1a, b). Physical examination showed some irregular, rugged scars on the facial 


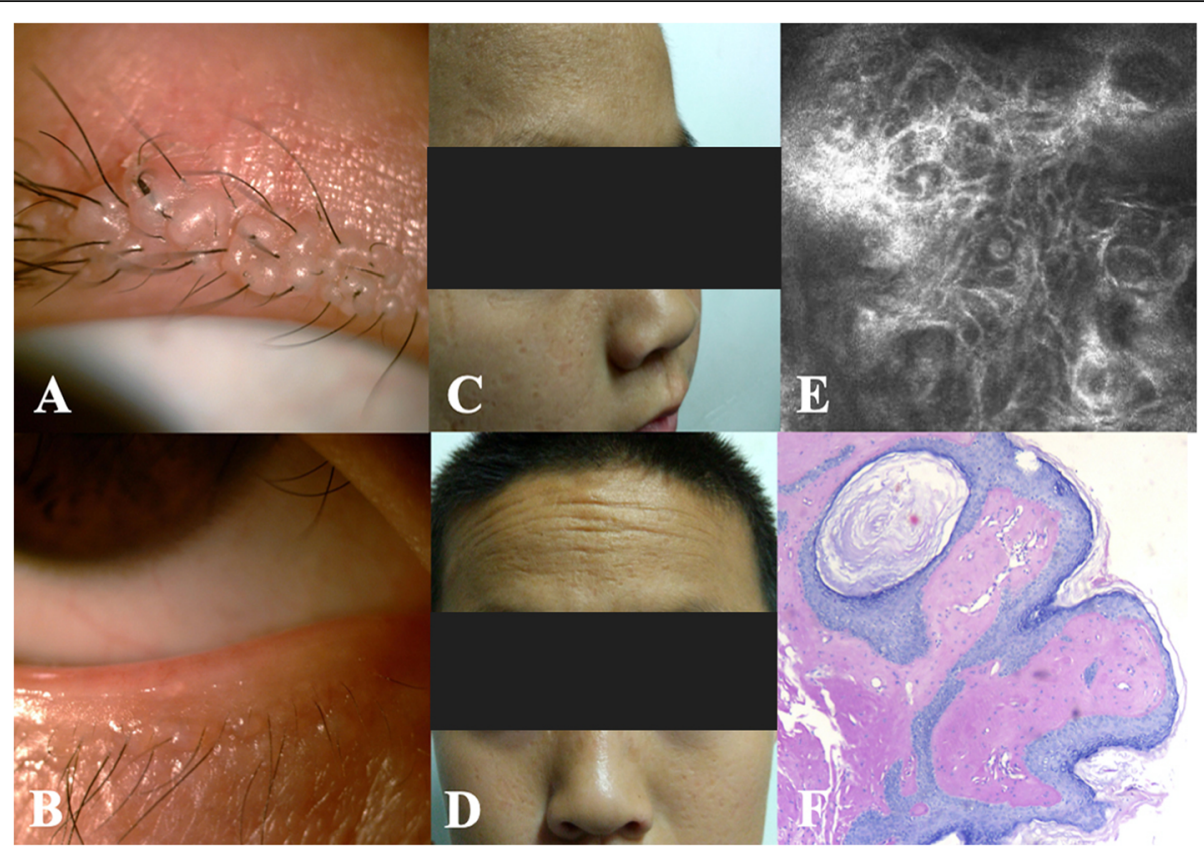

Fig. 1 Clinical features and histological findings of lipoid proteinosis in cases 1-2. $\mathbf{a}, \mathbf{b}$, waxy beaded papules on the margins of both eyelids and conjunctival congestion. $\mathbf{c}, \mathbf{d}$, irregular, rugged facial scarring and yellowish plaques and fine lines on the forehead. e, highly reflective fiber-like substance intricately arranged under the epithelial cells on in vivo confocal microscopy (800x). f, homogeneous eosinophilic hyaluronic material infiltrating around sweat glands and capillaries, associated with epidermal thinning (hematoxylin and eosin staining, 400x)

skin, yellowish plaques and fine lines on their foreheads (Fig. 1c, d). In vivo confocal microscopic (IVCM) analysis of the eyelids in case 1 showed highly reflective fibrous deposition under the epithelial cells (Fig. 1e).

A biopsy of an eyelid papule was performed in case 1 . Periodic acid-Schiff staining showed infiltration of an eosinophilic hyaluronic substance into many areas of connective tissue. Similar deposits were also found around sweat glands and capillaries, associated with epithelial thinning (Fig. 1f). Direct laryngoscopy combined with histologic examination showed epithelial dysplasia of the laryngeal and epiglottal mucosa.

Blood samples from the two affected cases and their parents were sent for gene sequence analysis. The two patients had the same homozygous CTG insert nucleotide 506 to 508 (c.506_508dupCTG) in exon 6 of the ECM1 gene (NM_004425.4) (Fig. 2a, b). Their parents had a similar heterozygous mutation at the same site, although they lacked the phenotype (Fig. 2c).

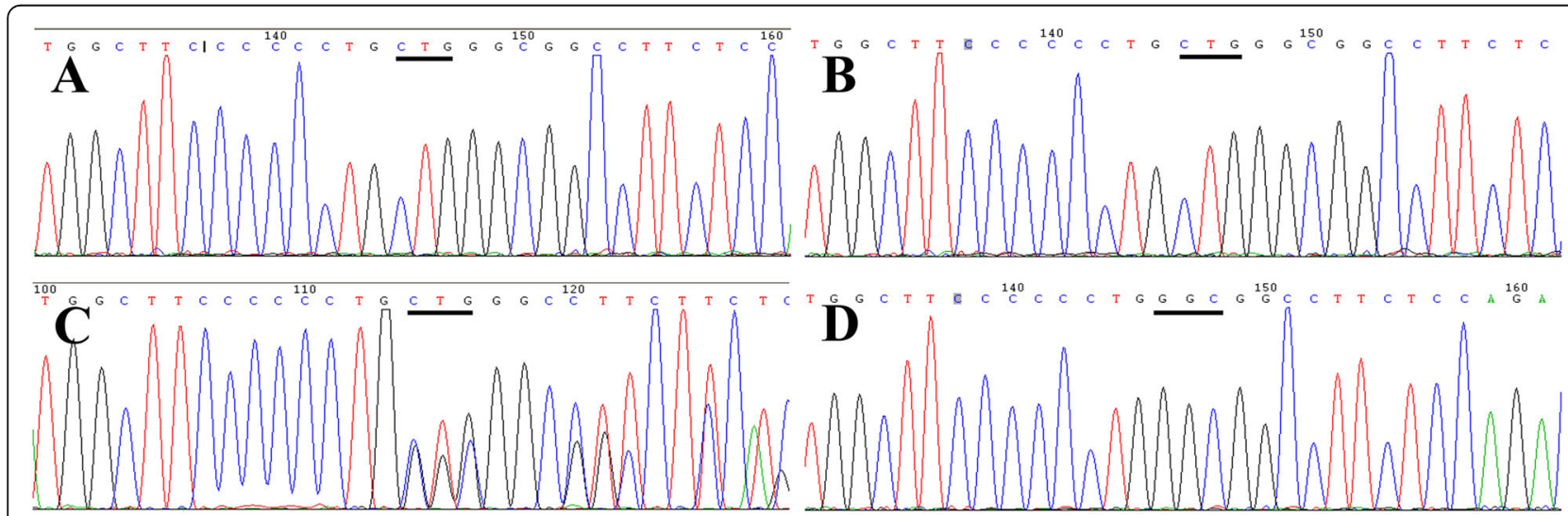

Fig. 2 Pedigree (case 1 \& 2) and sequencing results. a, b, homozygous CTG insert nucleotide 506 to 508 in exon 6 of the ECM1 gene in case 1 and case 2. c, same recessive heterozygous mutation in the parents. $\mathbf{d}$, wild type nucleotides in this portion of the DNA sequence 
The final diagnosis for the two boys was LP. The differential diagnosis included amyloidosis, hyalinosis, and erythropoietic protoporphyria. In response to the patient's symptoms, lubricant eye drops were prescribed. We also informed the patients that, if necessary, the papular eyelid lesions could be surgically removed.

\section{Case 3}

A 26-year-old woman who was bothered by the appearance of her eyelid margin with many beaded papules presented to our department. The hyperkeratotic papular lesions had appeared 15 years previously and worsened within the past 4 years. Her parents and close relatives had no similar history. The patient denied any history of prior surgery or medication.

The papular lesions of the upper and lower eyelids of both eyes were clustered closely together, forming beaded structures (Fig. 3a, b). In addition, she reported hoarseness since the age of 4 years. There was no history of similar symptoms among her family members. Cutaneous examination revealed waxy skin and erosive cutaneous lesions (Fig. 3c). Direct laryngoscopy showed deposition of a pale-yellow substance in the oropharynx and vocal cords, and movement of the vocal cords was restricted (Fig. 3d, e).

Genetic analysis showed a deleted T at nucleotide 507 (c.507deIT) in exon 6 of the ECM1 gene (NM_004425.4) (Fig. 4a). Analysis of the parents showed that this gene originated from her mother (Fig. 4c). Her father showed another suspected pathogenic gene in the same exon, with $\mathrm{C}$ replacing $\mathrm{T}$ at nucleotide $1174 \quad(\mathrm{c} .1174 \mathrm{C}>\mathrm{T})$ (Fig. 4b, d).

The woman's final diagnosis was LP. The differential diagnosis excluded amyloidosis, hyalinosis, and erythropoietic protoporphyria. In order to smooth the patient's ocular surface, we recommended lubricants. We advised her that if she wanted to improve her hoarseness, vocal cord surgery could be performed.

\section{Discussion and conclusions}

Lipoid proteinosis is a phenotypically heterogeneous condition with variable clinical features due to infiltration of the skin and multiple organs by a hyaline-like material. Patients typically present in early childhood with vocal hoarseness and skin and mucous membrane changes characterized by yellowish waxy areas affecting the face. The presence of a row of beaded papules along the eyelid margin, known as moniliform blepharitis, is also thought to be pathognomonic. Infiltration of the upper airway can induce dysphagia and, in severe cases, respiratory obstruction. Epilepsy and neuropsychiatric abnormalities may also appear in children [3].

Clinical diagnosis is confirmed by histological findings on biopsy of cutaneous and mucous membrane lesions, showing disruption and/or duplication of the basement membrane along with deposition of hyaline material at the dermo-epidermal junction, papillary dermis and surrounding capillaries, and around adnexal epithelia. We have provided an IVCM image, which has rarely been used to detect LP. The similarities between the biopsy and IVCM image may suggest an alternative method of detecting eyelid disease. In addition, head CT and MRI examinations are used to detect intracranial calcifications in severe cases.

The molecular basis of LP has recently been elucidated, and it appears to result from mutations in the gene encoding extracellular matrix protein 1 (Ecm1, ECM1; OMIM 602,201). ECM1 protein is expressed in the skin, mucosa, and several other tissues, including the placenta, heart, liver, small intestine, lungs, kidneys, and endothelial cells. ECM1 can stimulate blood vessel endothelial cell proliferation and influence the differentiation of keratinocytes. ECM1 promotes binding of collagen IV

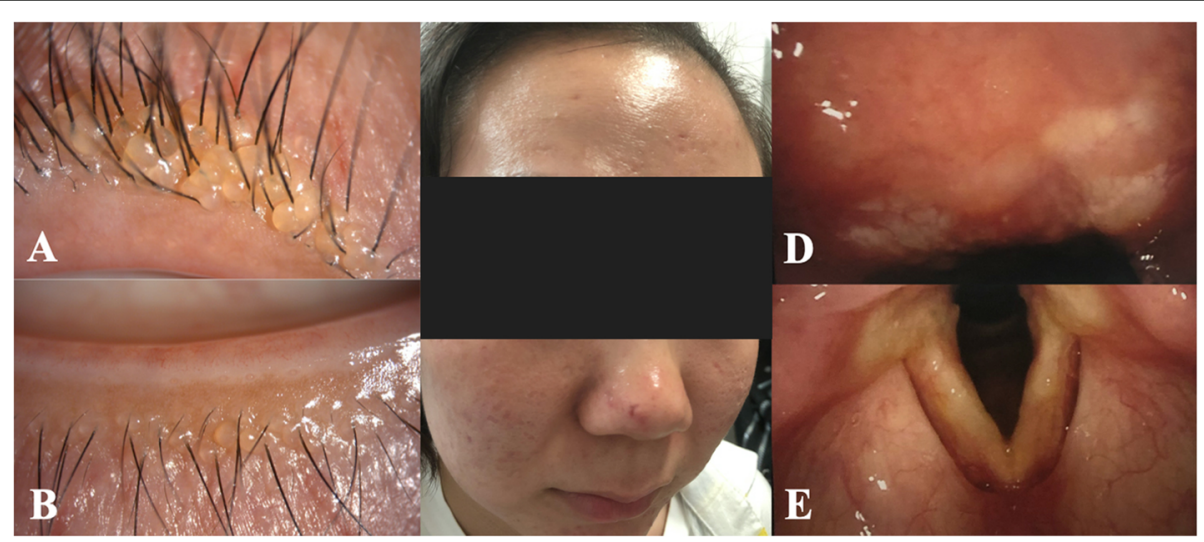

Fig. 3 Clinical features of lipoid proteinosis in case 3. a, b, beaded papules on the eyelids in case 3. c, multiple rugged facial scars. d, e, pale yellow substance deposition in the oropharynx and bilateral vocal cords 


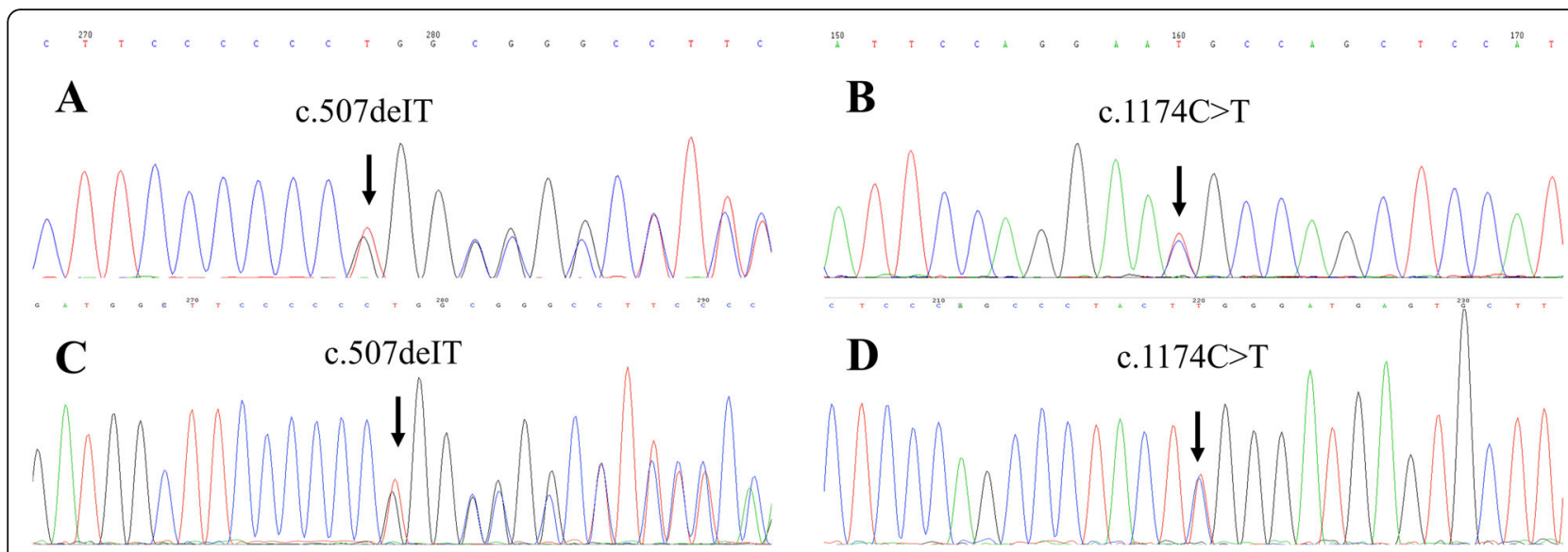

Fig. 4 Pedigree (case 3 and her parents) and sequencing results. a, b patient's genetic mutations: T base missing at nucleotide 507 and C base replacing $T$ base at nucleotide 1174 . c, the same genetic mutation was found in the patient's mother. $\mathbf{d}$, the same suspected pathogenic gene detected in her father

and laminin and acts as a biological glue by binding glycosaminoglycans to fibrillar protein growth factors [4]. Abnormal ECM1 function results in reduced production of normal collagen, with abnormal interactions with perlecan, MMP-9, fibulin, and laminin [5]. ECM1 protein may play a role in wound healing, scarring, and aging.

To date, at least 47 different mutations in the ECM1 gene have been reported in more than 50 unrelated patients with LP [6]. Frameshift and nonsense mutations have been described throughout the gene, with exons 6 and 7 being the most common locations. Mutations in these locations appear to have genotype-phenotype relevance. Patients with exon 7 mutations display slightly milder clinical features, while mutations in exon 6 (as in our cases) result in a more severe phenotype [7].

LP lacks effective treatment. Fortunately, with its slow progression and stable symptoms in adulthood, LP does not generally affect lifespan. It has been reported that oral dimethyl sulfoxide [8], D-penicillamine [8], and ettretinate [9] can alleviate symptoms involving the larynx and facial skin, but the efficacy of these treatments remains controversial. For skin lesions, skin treatments, avoidance of potential skin trauma to minimize scarring, and scar removal may be performed. To treat vocal hoarseness, the pale-yellow deposits on the surface and edges of the vocal cords may be removed surgically [10]. Ophthalmologists can make an early diagnosis of LP upon observation of the typical eyelid manifestations. If the disorder does not affect eyelid movement, the beaded papules along the eyelids should not be removed, and observation is the better option.

To conclude, when examining patients with eyelid papules, any voice or skin changes should be carefully noted. With a definite history of hoarseness, these patients should be sent for gene sequence analysis. If necessary, otorhinolaryngology and dermatology consults may help confirm the diagnosis. For LP, treatment is primarily symptomatic to improve patients' quality of life.

\section{Abbreviations}

LP: Lipoid proteinosis; ECM1: Extracellular matrix gene 1; IVCM: In vivo confocal microscopy

\section{Acknowledgements}

We thank the patients for their permission to publish their information.

\section{Authors' contributions}

ZW was the major contributor in writing the manuscript. AL made a substantial contribution to the revision of the manuscript. QL, the corresponding author, was primarily responsible for the experimental design and revision of the paper. The author(s) read and approved the final manuscript.

\section{Funding}

This work was supported by the Beijing Science and technology project (Z181100001918031), China. The funding organization played no role in the study design, patient recruitment or data interpretation.

\section{Availability of data and materials}

Data sharing is not applicable to this article as no datasets were generated or analyzed during the current study.

\section{Ethics approval and consent to participate}

This study was conducted at the Beijing Tongren Hospital with the approval of the hospital's Medical Ethics Committee (TRECKY2019-130). Written informed consent for gene test and participation in study was obtained from the study patients. For participants under 18 years of age, their parents confirmed the written informed consent instead.

\section{Consent for publication}

Written informed consent to publish personal or clinical details along with any identifying images was obtained from the study patients. And we got written informed consent from patient's parents, if participants were under 18 years old.

\section{Competing interests}

The authors declare that they have no competing interests.

\section{Author details}

'Beijing Key Laboratory of Ophthalmology and Visual Sciences, Beijing Institute of Ophthalmology, Beijing Tongren Eye Center, Beijing Tongren Hospital, Capital Medical University, 100005 Beijing, China. ${ }^{2}$ Quinze-Vingts National Ophthalmology Hospital, IHU FOReSIGHT, Paris, France. ${ }^{3}$ Versailles 
Saint-Quentin-en-Yvelines University, Versailles, France. ${ }^{4}$ Institut de la Vision, IHU FOReSIGHT, Sorbonne Université, INSERM, CNRS, 17 rue Moreau, F-75012

Paris, France.

Received: 20 May 2020 Accepted: 4 January 2021

Published online: 13 January 2021

\section{References}

1. Parmar NV, Krishna CV, De D, et al. Papules, pock-like scars, and hoarseness of voice. Lipoid proteinosis. Indian J Dermatol Venereol Leprol. 2013;79(1):136

2. Hamada T. Lipoid proteinosis. Clin Exp Dermatol. 2002;27(8):624-9.

3. $\mathrm{Xu} \mathrm{W}$, Wang $L$, Zhang $L$, et al. Otolaryngological manifestations and genetic characteristics of lipoid proteinosis. Ann Otol Rhinol Laryngol. 2010;119(11): 767-71

4. Zhang $R$, Liu $Y$, Xue $Y$, et al. Treatment of lipoid proteinosis due to the $p$. C220G mutation in ECM1, a major allele in Chinese patients. J Transl Med. 2014;12:85.

5. Mondejar R, Garcia-Moreno JM, Rubio R, et al. Clinical and molecular study of the extracellular matrix protein 1 gene in a spanish family with lipoid proteinosis. J Clin Neurol. 2014;10(1):64-8.

6. Callizo M, Ibáñez-Flores N, Laue J, et al. Eyelid lesions in lipoid proteinosis or Urbach-Wiethe disease: case report and review of the literature. Orbit. 2011; 30(5):242-4

7. Ravi Prakash SM, Verma S, Sumalatha MN, et al. Oral manifestations of lipoid proteinosis: A case report and literature review. Saudi Dent J. 2013;25(2):91-4.

8. Kaya TI, Kokturk A, Tursen U, et al. D-penicillamine treatment for lipoid proteinosis. Pediatr Dermatol. 2002;19(4):359-62.

9. Gruber F, Manestar D, Stasic A, et al. Treatment of lipoid proteinosis with etretinate. Acta Derm Venereol. 1996;76(2):154-5.

10. Xu W, Wang L, Zhang L, et al. Zhonghua Er Bi Yan Hou Tou Jing Wai Ke Za Zhi. 2010;45(4):301-4.

\section{Publisher's Note}

Springer Nature remains neutral with regard to jurisdictional claims in published maps and institutional affiliations.

Ready to submit your research? Choose BMC and benefit from:

- fast, convenient online submission

- thorough peer review by experienced researchers in your field

- rapid publication on acceptance

- support for research data, including large and complex data types

- gold Open Access which fosters wider collaboration and increased citations

- maximum visibility for your research: over $100 \mathrm{M}$ website views per year

At $\mathrm{BMC}$, research is always in progress.

Learn more biomedcentral.com/submissions 